\title{
Photokeratoscopy in fitting contact lens after penetrating keratoplasty
}

\author{
REIZO MANABE, MAMORU MATSUDA, AND TSUNEJI SUDA
}

From the Department of Ophthalmology, Osaka University Medical School, 1-1-50 Fukushima, Fukushima-ku, Osaka 553, Japan

SUMMARY The formula based on keratometric readings which is generally used is unsatisfactory for fitting contact lenses after penetrating keratoplasty, possibly owing to lack of information on the peripheral corneal toricity. In these studies a photokeratoscope was used to examine the entire graft topography. In all cases the corneal configuration became more normal after suture removal, but a considerable toricity still remained, especially near the graft-host junction. One month after removal of sutures spherical hard contact lenses (polymethylmethacrylate, PMMA) were fitted to 30 patients in accordance with data obtained by computerised analysis of the photokeratograms. Of the 30 patients (contact lens wearers) $27(90 \%)$ obtained a stable vision of better than $20 / 30$ for eight hours daily, and $24(80 \%)$ achieved a stable vision of $20 / 20$ for their full waking hours. After one year the contact lens wearers showed a significant decrease in the extent of astigmatism when compared with the non-contact-lens wearers (10 patients). These results suggest that the photokeratoscope can be more useful than ordinary keratometers in fitting contact lenses after keratoplasty, and that hard contact lenses have moulding effects on the graft topography.

Improvements in surgical techniques and postoperative care have resulted in a higher rate of clear grafts after penetrating keratoplasty. However, there are a considerable number of cases with a high degree of corneal astigmatism and ametropia, which prevent good vision. Although surgeons attempt to obtain emmetropic anastigmatic transplants, no reliable reproducible techniques have yet been established. ${ }^{1-5}$

Because most postkeratoplasty patients have some degree of corneal irregular astigmatism, contact lenses, especially hard contact lenses, are required for satisfactory visual rehabilitation. ${ }^{6}$ However, conventional methods for fitting contact lenses, based on keratometric readings, do not apply to these patients. ${ }^{7}$ Ordinary keratometers provide only measurements of the apical corneal curvatures approximately $2 \mathrm{~mm}$ from the visual axis, while a higher degree of toricity is often present near the host-graft junction. To obtain successful results good

Correspondence to Mamoru Matsuda, MD, Department of Physiology, Medical College of Wisconsin, 8701 Watertown Plank Road, Milwaukee, WI 53226, USA. Reprint requests to Reizo Manabe, MD, Department of Ophthalmology, Osaka University Medical School, 1-1-50 Fukushima, Fukushima-ku Osaka 553, Japan. relationships between a lens and the central and peripheral cornea are of great importance. Therefore it can be said that the lack of information on the peripheral corneal toricity may be closely related to difficulties in fitting contact lenses after keratoplasty.

In this study the entire corneal topography was examined with a photokeratoscope, and hard contact lenses were fitted in accordance with the data obtained by computerised analysis of the photokeratograms. ${ }^{8}$

\section{Patients and methods}

Forty patients with unilateral phakic corneal transplants were studied. The preoperative diagnoses of these patients included keratoconus ( 25 eyes), herpes simplex stromal keratitis (8 eyes), and leucoma of unknown aetiology ( 7 eyes). None had evidence of retinal diseases, including age-related macular degeneration and clinically significant cystoid macular oedema. The ages of the patients ranged from 15 to 75 years (mean 47 years). Hard contact lenses (polymethylmethacrylate, PMMA) were fitted to 30 patients (contact lens wearers), while lenses were not prescribed for the other 10 patients (non- 
contact-lens wearers) because they could not manipulate the lenses owing to poor dexterity. The latter group were used as controls in a study of the effect of contact lens wear on postkeratoplasty astigmatism.

All keratoplasties were performed by one surgeon (RM) under local anaesthesia. The corneal button was removed from the epithelial side with a trephine ( 7 to $8.5 \mathrm{~mm}$ in diameter) and secured in place with eight interrupted 9-0 virgin silk sutures followed by a single $10-0$ nylon monofilament running suture. ${ }^{910}$ After reformation of the anterior chamber of the eye with a balanced salt solution, the interrupted sutures were removed. The running suture was removed six to 12 months (mean 10 months) postoperatively. All corneas were serially examined with a photokeratoscope (SUN PKS-1000) which measures the entire corneal topography (Fig. 1). The photokeratograms obtained were subjected to a computerised analysing system $^{8}$ that calculated the entire corneal configura- tion as well as the keratometric readings" (Figs. 2 and 3 , middle). In the corneas where the mire images were out of focus owing to highly irregular or extensive curvatures the midpoint of the blurred mire images was analysed to calculate the average keratometric readings.

Contact lenses were fitted in accordance with the data obtained by photokeratoscopy and refraction of the eye. This procedure was done at one month after suture removal, during which the corneal epithelial defects associated with the suture removal were completely healed in all the cases studied. Spherical contact lenses with a diameter of at least $1 \mathrm{~mm}$ larger than the grafts were fitted in order to minimise damage to the host-graft junction. An acceptable fit was considered to be a lens with good centration and an adequate movement ( 1 to $2 \mathrm{~mm}$ ) during blinking to obtain proper tear exchange under the lens. The proper tear exchange was designated as a washout of fluorescein occurring in less than five minutes. The

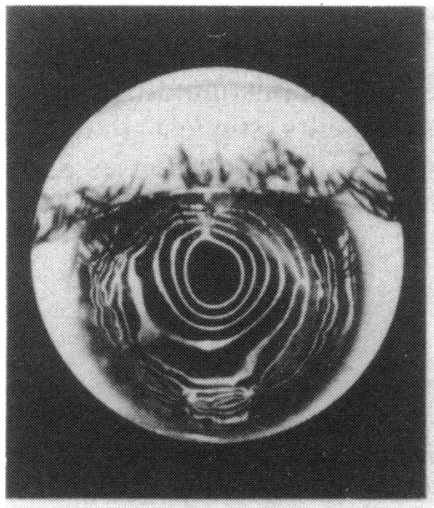

Post op. $7 \mathrm{M}$

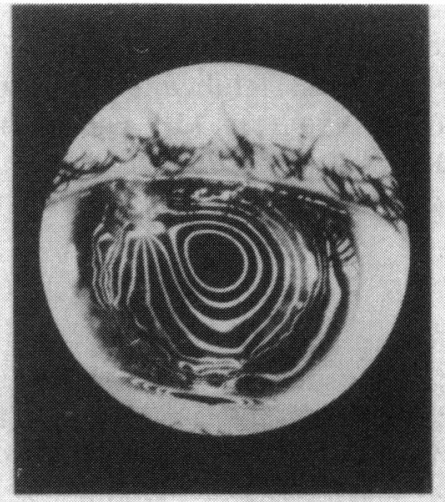

Post op. $12 \mathrm{M}$

Before suture removal

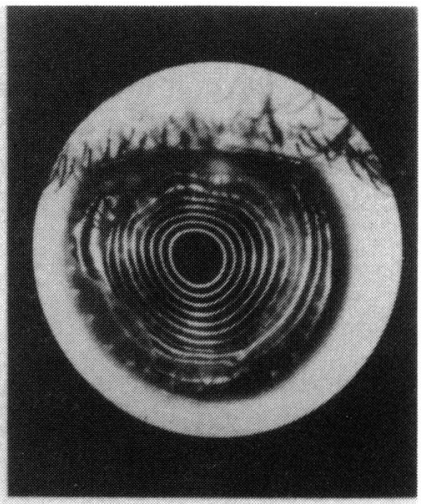

Post op. $14 \mathrm{M}$

After suture removal
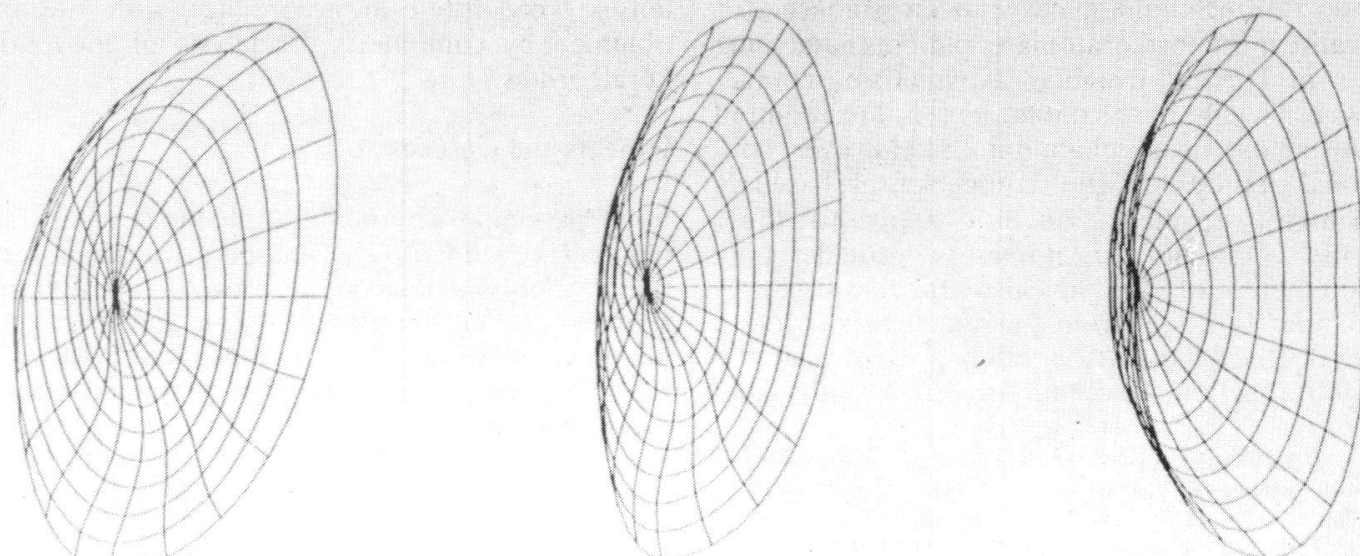

Fig.1 Sequential photokeratograms after penetrating keratoplasty. 

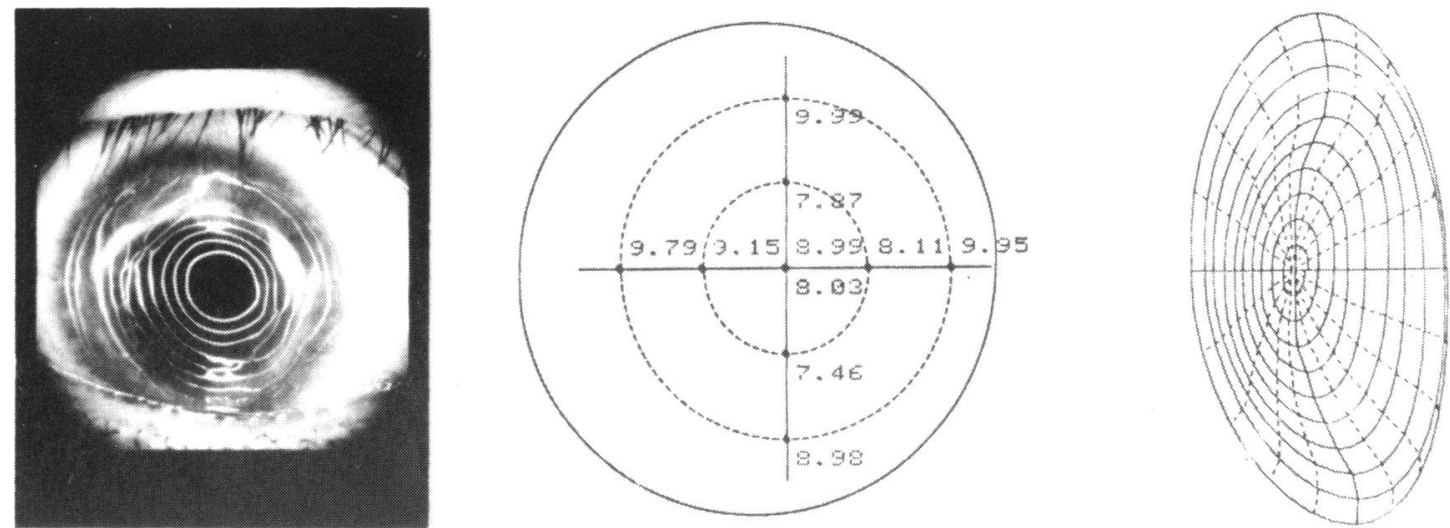

Fig. 2 The corneal topography in a 41 -year-old male with herpes stromal keratitis who underwent keratoplasty with an 8 mm corneal button. Each number in the middle figure represents the radius $(\mathrm{mm})$ of the curvature for the respective region of the cornea, which provided quantitative parameters to fit a lens.

peripheral posterior curve of the lens was modified according to the peripheral corneal toricity, if needed, in order to obtain a better lens-cornea relationship.

\section{Results}

In all the cases studied the corneal topography was quite flat and irregular during the early period after penetrating keratoplasty (Fig. 1, left), but it became steeper and more regular with time (Fig. 1, middle). Suture removal resulted in sudden changes in the graft topography (Fig. 1, middle and right). There was considerable individual variation in the degree of these topographic changes. After suture removal the configuration of the central cornea became more spherical, but a considerable toricity still remained, especially near the host-graft junction (Fig. 1, right).
Fig. 2 shows the corneal topography in a 41-yearold male with herpes stromal keratitis who underwent keratoplasty with an $8 \mathrm{~mm}$ corneal button. Because the cornea had flatter than normal central and peripheral curvatures ${ }^{12}$ (Fig. 2, middle), a lens with a diameter of $9.2 \mathrm{~mm}$ and a central posterior (base) curve of $8.65 \mathrm{~mm}$ was fitted, and the peripheral posterior curve of the lens was modified to be flatter. This patient has been wearing the lens for his full waking hours with a stable vision of 20/20.

Fig. 3 shows the corneal topography in a 24-yearold female with keratoconus undergoing keratoplasty with a $7.5 \mathrm{~mm}$ graft. Because the cornea had steeper than normal central curvatures and flatter than normal peripheral curvatures ${ }^{12}$ (Fig. 3, middle), a lens with a diameter of $9.5 \mathrm{~mm}$ and a base curve of $7.35 \mathrm{~mm}$ was fitted and the peripheral posterior curve of the lens was also modified to be flatter. The patient
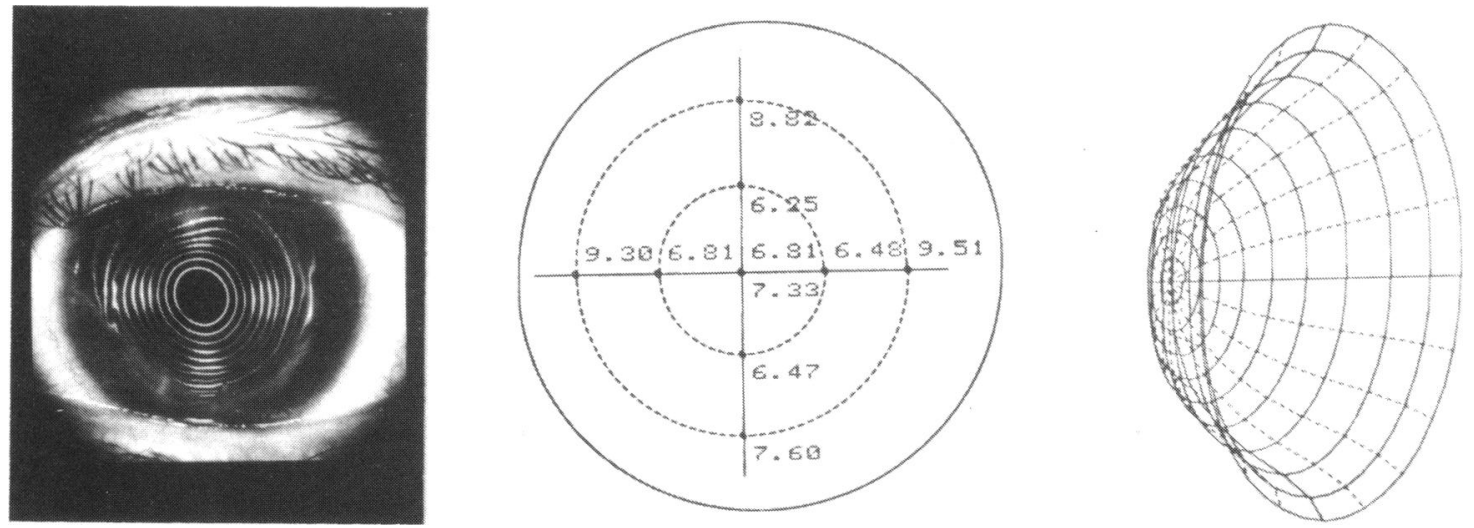

Fig. 3 The corneal topography in a 24-year-old female with keratoconus who underwent keratoplasty with a $7.5 \mathrm{~mm}$ graft. Each number in the middle figure represents the radius $(\mathrm{mm})$ of the curvature for the respective region of the cornea, which provided quantitative parameters to design a lens. 
Table 1 Changes in corneal astigmatism

\begin{tabular}{lllll}
\hline $\begin{array}{l}\text { Contact lens } \\
\text { wear }\end{array}$ & \multicolumn{4}{l}{ Average astigmatism (dioptres) } \\
\cline { 2 - 5 } & $0^{*}$ & 3 months & 6 months & 12 months \\
\hline Yes $(\mathrm{n}=30)$ & $4 \cdot 5$ & $2 \cdot 7$ & $2 \cdot 5$ & $2 \cdot 2$ \\
No $(\mathrm{n}=10)$ & $4 \cdot 2$ & $4 \cdot 0$ & $3 \cdot 8$ & $3 \cdot 5$ \\
& $\mathrm{NS}$ & $\mathrm{NS}$ & $\mathrm{NS}$ & $\mathrm{p}<0.05$ \\
\hline
\end{tabular}

* Corresponds to one month after suture removal.

NS= not significant (Student's $t$ test).

has worn the lens for 10 hours a day with a stable vision of 20/20.

With contact lens correction alone $27(90 \%)$ of the 30 patients obtained a stable vision of better than $20 / 30$ for eight hours a day, and $24(80 \%)$ obtained a stable vision of $20 / 20$ for their full waking hours. Only three patients could not obtain either more than eight hours' lens wear or a stable vision of better than $20 / 30$. All of them had an astigmatism of greater than 6 dioptres (D).

So far as the lens fitting and complications were concerned, a small number of problems were observed. Inadequate centration and/or movement of the lens was noted in three patients $(10 \%)$, all of whom had a greater than $6 \mathrm{D}$ astigmatism and also showed an excessive attraction of the lens for the cornea. Three patients $(10 \%)$ had an air bubble under the lens, which did not interfere with tear exchange or obscure vision. Six patients $(20 \%)$ showed a slight degree of ocular congestion, and 4 $(13.3 \%)$ occasionally developed a mild degree of fluorescein staining of the corneal epithelium. None of these complications resulted in discontinuing the use of the lens.

The average astigmatism prior to contact lens wear (one month after suture removal) was 4.5 $\mathrm{D}$ for the contact lens wearers and 4.2 D for the non-contactlens wearers respectively (Table 1 ). There was no significant difference between these values. The noncontact-lens wearers showed a gradual decrease in the astigmatism during the one year follow-up. This decrease, however, was not statistically significant. By comparison, the contact lens wearers showed a marked decrease in the astigmatism to $2.7 \mathrm{D}$ during the first three months and a gradual decrease thereafter. After one year the average astigmatism for the contact lens wearers $(2 \cdot 2 \mathrm{D})$ was significantly smaller $(p<0.05)$ than that for the non-contact-lens wearers (3.5 D; Student's $t$ test).

\section{Discussion}

Even if carried out by skilled surgeons with modern techniques most corneal grafts result in some degree of ametropia and irregular astigmatism,,-1 and con- tact lenses are often required for satisfactory correction of vision. ${ }^{67}$ Because of the variation in the extent of corneal toricities from patient to patient, fitting contact lenses after keratoplasty is usually made by trial and error. The fitters must be skilled to modify the parameters of the lens to effect proper centration, venting, and movement, and many lenses with various base curves and diameters may need to be tried. ${ }^{6}$ Most of these tedious procedures could be attributed to incomplete information on the entire graft topography, since ordinary keratometers provide only the central curvatures approximately $2 \mathrm{~mm}$ from the visual axis.

In contrast the photokeratoscope used in this study has the advantage of permitting examination of the entire corneal topography, which has been shown to be more useful than ordinary keratometers in fitting contact lenses in patients with some degree of refractive error ${ }^{8}$ and those with keratoconus. ${ }^{1314}$ Our study showed that this instrument can also be useful in fitting contact lenses after penetrating keratoplasty.

Our serial observations on the graft topography showed that, although the central corneal configuration becomes more normal after suture removal, a considerable toricity still remains, particularly in the peripheral cornea. This aspect appeared to be significantly related to the difficulties in the fitting, because most of our cases required modification of the peripheral posterior curves of the lens to obtain good centration and an adequate lens-cornea relationship. These modifications, however, were made easily from the photokeratoscopic data for the peripheral corneal toricity.

All the lenses used in this study were larger than the grafts. Lenses smaller than the grafts can be fitted, but because of their small size they are usually uncomfortable and unstable, causing fluctuating vision and possible damage to the host-graft junction. ${ }^{6}$ In order to stabilise such smaller lenses, steepening the base curve is often required, which would increase the attraction of the lens for the cornea, resulting in intolerance of the lens after several hours of lens wear. By comparison larger lenses may decrease the damage to the host-graft junction ${ }^{67}$ and offer good centration and movement, and they may provide a stable vision and a proper venting which facilitates oxygen exchange under the lens. ${ }^{7}$ Contact with the upper lid can also help to provide good stabilisation and movement of the lens.

Despite the satisfactory results in most of our series, three patients with an astigmatism of greater than $6 \mathrm{D}$ did not obtain a vision of better than 20/30 and did not tolerate the lens for more than eight hours a day. Such patients would require toric lenses rather than spherical ones ${ }^{6}$ or could be considered for 
refractive surgery, such as relaxing incisions or wedge resection. ${ }^{15}$

As reported previously, ${ }^{616}{ }^{17}$ we found that the graft topography is in a state of transition for several months after suture removal. We also found prospectively that the contact lens wearers have a significant decrease in the extent of corneal astigmatism after one year of lens wear when compared with the noncontact-lens wearers. A similar observation has been made in a retrospective study by Ruben and Colebrook. ${ }^{17}$ These findings indicate that contact lenses have a moulding effect on the graft topography. ${ }^{617}$

We thank Mr Etsuji Adachi (SUN Contact Lens Co. Ltd., Kyoto, Japan) for his valuable technical assistance in this study.

\section{References}

1 Troutman RC, Meltzer M. Astigmatism and myopia in keratoconus. Trans Am Ophthalmol Soc 1972; 70: 265-77.

2 Troutman RC. Microsurgical control of corneal astigmatism in cataract and keratoplasty. Ophthalmology (Rochester) 1973; 77: 563-72.

3 Troutman RC, Kelly S, Kaye D, Clahane AC. The use and preliminary results of Troutman surgical keratometer in cataract and corneal surgery. Ophthalmology (Rochester) 1977; 83: 232-8.

4 Troutman RC. Astigmatic considerations in corneal graft. Ophthalmic Surg 1979; 10: 21-6.

5 Troutman RC, Gaster RN. Surgical advances and results of keratoconus. Am J Ophthalmol 1980; 90: 131-6.
6 Daniel R. Fitting contact lenses after keratoplasty. Br J Ophthalmol 1976; 60: 263-5.

7 Garcia GE. Contact lens fitting in keratoconus and following keratoplasty. In: Leibowitz HM. Corneal disorders. Philadelphia: Saunders, 1984: 678-82.

8 Kuyama H, Sasamoto K, Maruyama S, Itoi M. A new photokeratoscope for contact lens in clinic. J Jpn Contact Lens Soc 1979; 21 : 80-4.

9 Matsuda M, Suda T, Manabe R. Long-term observations on the graft endothelium with different postoperative courses. Jpn J Ophthalmol 1983; 27: 556-66.

10 Matsuda M, Suda T, Manabe R. Serial alterations in endothelial cell shape and pattern after intraocular surgery. Am J Ophthalmol 1984; 98: 313-9.

11 Matsuda M, Suda T, Manabe R. Quantitative analysis of endothelial mosaic pattern changes in anterior keratoconus. Am J Ophthalmol 1984; 98: 43-9.

12 Hasegawa $\mathrm{S}$, Masuda $\mathrm{N}$, Terao $\mathrm{N}$, Inatomi A. Corneal topography using the photokeratoscope. J Jpn Contact Lens Soc 1981; 23: 208-14.

13 Suda T, Matsuda M, Manabe R, Adachi E. Evaluation of the fitting of hard contact lenses for keratoconus. J Jpn Contact Lens Soc 1982; 24: 84-94.

14 Maruyama S, Suda T, Inoue Y, Matsuda M. Computerized contact lens designing system for keratoconus. J Jpn Contact Lens Soc 1983; 25: 185-90.

15 Krachmer JH, Fenzel RE. Surgical correction of high postkeratoplasty astigmatism. Arch Ophthalmol 1980; 98: 1400-2.

16 Matsuda M, Suda T, Manabe R. Chronological changes of graft topography after penetrating keratoplasty. Folia Ophthalmol Jpn 1981; 32: 1347-52.

17 Ruben, M, Colebrook E. Keratoconus keratoplasty curvatures and contact lens wear. Br J Ophthalmol 1979; 63: 268-73.

Accepted for publication 25 April 1985. 